\title{
Supporting Context-Aware Collaborative Learning Activities by CAFCLA
}

\author{
Óscar García, Ricardo S. Alonso, Dante I. Tapia, and Juan M. Corchado
}

\begin{abstract}
The integration of Information and Communication Technologies (ICT) in daily life has improved the learning process by means of context-aware technologies. Through the use of technology, new ways of learning has emerged allowing to become the learning process more ubiquitous. However, it is necessary to develop new tools that can be adapted to a wide range of technologies and different application scenarios. This paper presents CAFCLA, a framework that allows developing context-aware learning applications. CAFCLA integrates different context-aware technologies, so that learning applications designed, developed and deployed upon it are dynamic, adaptive and easy to use by users such as students and teachers.
\end{abstract}

Keywords: Computer Supported Collaborative Learning, Context-Aware Learning, Real Time Location Systems, Wireless communication.

\section{Introduction}

The society has been flooded with a wide range of different mobile devices which user interfaces or communication skills are improved day by day [1]. Thanks to these advances, we are surrounded by technology that has changed our habits and customs [2] by receiving information from multiple sources. The ability of the devices to collect, offer and react to the information from the environment is the main topic in Context-aware Computing [3].

The usage of Information and Communication Technologies (ICT) has been present in educational innovations over recent years [4], modernizing the traditional transmission of contents through electronic presentations, email or more complex learning platforms such as Moodle and fostering collaboration between

Óscar García · Ricardo S. Alonso · Dante I. Tapia · Juan M. Corchado

Department of Computer Science and Automation, University of Salamanca.

Plaza de la Merced, s/n, 37008, Salamanca, Spain

e-mail: \{oscgar, ralorin, dantetapia, corchado\} @usal.es 
students (Collaborative Learning) [5]. Beside the use of those general-purpose tools in education, other tools that make more specific use of technology have appeared. This applies to those that make use of Context-awareness information and ubiquitous computing and communication.

The inclusion of context-awareness in educational scenarios and processes refers to Context-aware Learning [6], a particular area of application of Context-aware Computing [3]. Moreover, the ability to characterize and customize the context that surrounds a learning situation at a certain time and place provides flexibility in the educational process. This way, learning does not only occur in classrooms, but also in a museum, park or any other place [7], obtaining ubiquitous learning spaces. Thus, there is an extensive literature that addresses the problem of this kind of learning, highlighting those works that attempt to solve contextual information acquisition and providing data to users [8]. The use and integration of different technologies and the approach to specific learning activities characterize these solutions. However, the complexity of understanding and use of the technology and solutions in the aforementioned works does not allow a wide use of them.

This paper presents the use of CAFCLA [9], a framework aimed at designing, developing and deploying context-aware educational scenarios. Teachers are able to characterize the context where the learning activity will occur through the creation of a world model in which locate data collectors (e.g., sensors), identify and characterize areas of interest (e.g., paintings in a museum), etc. Moreover, the collaboration between students and the customization of the information available is also provided and can be integrated in the activity design. For that, a botanical garden has been chosen as scenario, as it presents the physical and content features to develop a context-aware collaborative learning activity: indoor and outdoor spaces within multiple learning objects (trees and flowers).

The following section describes the background and problem description related to the presented approach. Then, CAFCLA are described: what kinds of activities are covered, how the context of the activity can be defined, who the users are, which activities are implemented by the framework, taking a botanical garden as example scenario. Finally, the conclusions and future work are depicted.

\section{Background and Problem Description}

A growing interest in educational software, commonly known as e-learning, has appeared over recent years [5]. Mobile devices provide important benefits to education: mobility, communication skills including collection and provision of contextual information, as well as precise location anytime. Mobile Learning is defined as "the processes of coming to know through conversations across multiple contexts amongst people and personal interactive technologies" [10]. This definition implies two important ideas: first of them is that technology can be involved into the learning process; the second idea suggests that mobile learning emphasizes the communication between the involved people and their interaction with the context [11]. 
Mobile devices allow learners to work outside the classroom to enhance collaborative learning both indoor (e.g., museums) or outdoor (e.g., parks) spaces that present any didactic interest [12]. New mobile devices are equipped with features that facilitate the acquisition of contextual information and location. Contextual information includes any data that can be used to characterize a person, place or object that is considered relevant to the interaction between users, between user and applications or systems, or even between systems and applications [3]. In addition to the relevant information that context provides, it is important to consider other parameters that relevantly affect this type of information, such as identification, time and location [3]. The information exchange taking place between technology and users, in order to contextualize an environment in which learning takes place, and customize the content of the learning activity can be understood as collaboration. Thus, Context-aware Learning must take into account the interactions between people and the different technological components of the system in all its combinations.

Providing contextual information and fostering collaboration between students benefit the learning process [1]. Moreover the combination of Collaborative and Context-aware Learning naturally leads to thinking about ubiquitous learning spaces, characterized by "providing intuitive ways for identifying right collaborators, right contents and right services in the right place at the right time based on learners surrounding context such as where and when the learners are (time and space), what the learning resources and services available for the learners, and who are the learning collaborators that match the learners' needs" [13].

Attending to different technologies there are several trends focused on provide context information to the learning process. A first approach to provide contextual information is "tagging the context". RFID (Radio Frequency IDentification) is the most spread technology [14]. Its use is tedious because teachers have to tag any object they would like to characterize. GPS (Global Positioning System) is the most used technology to provide location in Context-aware Learning [15]. This location system provides a high accuracy level and is currently implemented in a wide range of smart phones and mobile devices. In those cases, the mobile device provides a position to the system. Those solutions are used in different scenarios such as route planning [15]. However, most of those works implement specific applications, but do not propose a general purpose model in which GPS technology is included to facilitate the provision of contextual data. Moreover, teachers cannot decide the activity they would like to implement.

Indoor environments are very common in learning: museums, laboratories or the school are places where activities that require mobility can be developed. Trying to cover this lack, different location systems based on Active RFID [15] or Wi-Fi [16] are used. Both cases the performance of systems is similar: student's position is determined by the access point which is providing coverage in each moment. This type of approach has significant limitations when developing context-aware learning activities: the location accuracy is too poor so teachers are not able to characterize small areas or individual items. This situation presents an 
important problem when areas where context information is different are close (e.g., two paintings in a museum).

The review of the literature evidences some lacks in the Context-aware Learning systems proposed until now. Even some works try to combine different technologies to cover as much situations as possible [16], teachers are not able to choose different activities to implement or feel free to determine collaborations between students. Moreover, the technology complexity is not transparent to teachers, who are responsible to design the learning activity. Those solutions are focused on the technological part of the problem and do not take into consideration what teachers should do to design the learning process. Next section presents an example of use of CAFCLA that illustrates how this framework takes into consideration the teachers' point of view when designing a context-aware collaborative learning activity.

\section{CAFCLA Scenario}

CAFCLA (Context-Aware Framework for Collaborative Learning Applications) is a framework focused on the design, development and deployment of collaborative learning applications that make use of contextual information [9]. CAFCLA involves multiple users and characterizes each one according to their role in the design, development, deployment and implementation of a learning activity. Moreover, CAFCLA takes into consideration all aspects surrounding the whole learning process design. These aspects include the objectives or goals that students must reach, the contents of the learning activity, the teaching resources available, the physical or virtual spaces selected or the assessment and activity monitoring. All these aspects do not only involve the teacher, but there is also a technical component that must be undertaken by staff that sometimes do not present an education profile. More specifically, three different roles can be identified in the process of design and development of activities considered in this work.

On first place, teacher is responsible for designing the activity that will be deployed using CAFCLA. Some of the task that teachers carry out includes defining which students participate in the activity, what kind of activity is carried out, which collaborations between students are allowed, which areas and objects of interest are described in the activity, which are the objectives of the activity and which is the data that the system will store to be provided during the activity. Then, developer makes use of all the tools provided by CAFCLA (analysis and design, programming, etc.) to develop the application that students will use, according to the activity designed by teachers. Finally, technician is responsible for deploying the technology infrastructure needed to carry out the activity developed using CAFCLA, following the premises and recommendations set by the framework.

Moreover, student is the participant who finally conducts the activity designed by the teacher. They can access the resources offered by the application through different devices selected for that purpose. In addition, they are able to collaborate 
between them to achieve the objectives of the activity. Their performance follows the rules set by the teacher at all times.

Once CAFCLA users have been defined, the way in which contextual information is organized is described. According to AmI premises, CAFCLA emphasizes on technological transparency and ease of use for both students and teachers. Contextual information is closely related to the environment where the activity takes place, so any place or item can provide relevant information to be used in the learning process. Thus, teachers are able to describe any place or item relevant to the activity regardless of size and location. In order to better structure contextual information, three description levels have been defined, so that the information can be provided with the granularity required by the activity.

- Scenario: It represents the physical space where the activity will be deployed. To better illustrate the explanation a botanical garden has been chosen to deploy a collaborative learning activity. This scenario consists of an outdoor enclosure where different species of trees, shrubs and flowers grow. Furthermore, in the center of the enclosure there is a greenhouse where multiple flower species grow. In this case the scenario is the botanical garden and it could be divided into two sub-environments: the first one that includes all the study to be performed in the greenhouse (indoor plants), and the second, which would cover the rest of the botanical garden, including all the growth area of outdoor plants.

- Area of interest: Different areas that determine spaces in which a relevant part of the activity will take place. These areas include a physical space where one or several goals of the activity should be reached. The teacher is responsible for identifying, locating and making relevant contextual characterization into them. The areas of interest provide contextual information to the students in the way that the design made by the teacher shows. Continuing the example of the botanical garden, in the external environment three different species of trees grow: pine, oak and poplar. In this case the teacher can create four areas of interest: three individual areas covering spaces where trees grow and a fourth area which is the greenhouse. For each of them, the teacher defines the physical space that it delimits. It also includes a description of each area, based on the design of the activity, that is given to students.

- Object of interest: in the same way that the environments in which the scenario is divided in different areas of interest, within these areas can be included several specific objects that are interesting to the learning activity. Teachers follow the same procedure as in previous cases, since they are responsible for identifying, locating and characterizing these objects. In the example of the botanical garden there may be multiple objects of interest within each of the areas of interest. For example, in the greenhouse grow a wide variety of flowers, and each kind may be an object of interest so teachers are able to identify, place and characterize each one into the greenhouse. 
The activities that can be deployed using CAFCLA is another important part of the framework implementation. Different collaborative activities has been evaluated to be integrated by CAFCLA and three of them have been selected: "Treasure Hunting", "Collaborative WebQuest" and "Jigsaw". Different criteria have been taken into account to choose these activities. First of all, these activities can be deployed anywhere and anytime. Secondly, collaboration is possible to be included in all of them. Thirdly, the activities allow teachers to create a learning process that can be monitored and modified at all times. Fourthly, the participants of these activities can be divided into different groups. And finally, all of them can include different routes or physical paths to be followed by the students. However, CAFCLA is an open framework that is able to integrate any other activities that teachers may consider in the future.

Depending on the chosen collaborative activity, the teacher can add the necessary data to complete the learning process. More specifically, the requirements to be considered for each of the activities that are included are described as follows. First activity implemented is a Treasure Hunt. In this activity the teacher can create working groups that are assigned to the corresponding students and determines which devices are used by each students' group. Students that form a group are able to collaborate with each other at all times. Furthermore, the teacher can define different routes that students must follow to uncover clues and collect information. After setting the scenario, the areas of interest and the objects of interest, the teacher defines each route on the map, and indentifies which are the clues given to each group. Routes do not have to be composed of one only path, but may include branches that allow the division of tasks between the different students that are part of the group. The teacher can assign a path or more to a particular group and also may indicate which tracks are key, so that the students are required to complete a milestone to continue receiving information. Finally, the teacher defines a challenge or final question that must be completed or answered with the information received on each track, such as a questionnaire, a document or a presentation to be made.

Collaborative WebQuest is the second activity implemented. The process of defining and describing the scenario, the areas and the objects of interest, the users and the groups of students is common to all the activities. In this activity the teacher is able to design a battery of questions to be answered in each area of interest. Questions can be presented to be answered in a written way or as a test which offers different response options. These questionnaires can be tailored to each users' level and several can be defined for the same area or object of interest. In addition, the teacher can create a final questionnaire to be performed at a particular location (e.g., the classroom) which summarizes all questions regarding the questionnaires that have been made in the activity. Likewise, the teacher has the capacity to define different working groups formed by the number of students it may consider, assigning devices that each group or student uses. Likewise, the teacher indicates the questionnaires that each group must respond. The onset of 
the activity is performed for each group in the particular zone determined by the teacher through a first task in explanation of the activity.

The last implemented activity is a Jigsaw. As in the previous activities, the process of defining and describing the scenario, the areas and the objects of interest, the users and the groups of students is the first step to be completed. In this activity the teacher divides the activity into different subjects to study individually and then in different groups. First, the teacher assigns each student a specific topic in which will be "expert". Later, two different types of groups are formed: on the one hand by students who have been assigned with different topics (each group will consist of an expert in each of the different themes described) and on the other hand by "expert" students in the same topic. Similarly, the teacher determines which of the areas of interest that have been created belongs to each of the assigned topics. For the proper operation of the activity, the teacher indicates what documents will be generated at each of its stages: single phase, experts phase (collaboration among students working under the same topic) and group phase (collaboration among students working on different topics). The final result of the activity (e.g., a presentation) should be exposed by the group's leader, a role that is assigned by the teacher.

\section{Conclusions and Future Work}

The use of Information and Communication Technology in the different areas has increased in recent years thanks to the emergence in society of mobile devices, easy access to currently existing technology and the many features they present, such as communication protocols and context-aware technologies. However, it is difficult to develop applications to squeeze all the potential offered by technology, especially when the main objective is the development of technological applications that are transparent to users.

CAFCLA has been designed with the objective to design and develop a set of tools that provide a basis for designing, developing and implementing contextaware collaborative learning activities. CAFCLA is a framework that integrates different context-aware technologies, such as Real Time Locating Systems, and several communication protocols that abstract educators and developers of context-aware collaborative learning activities from the complexity of the use of different technologies simultaneously. In this case, CAFCLA focuses on provide a set of tools and methods to teachers, developers and technical staff in order to easily design, develop and deploy this type of learning activities.

The development of the framework has been approached from the teacher's point of view. Thus, the design of different kind of collaborative activities has been implemented by CAFCLA focusing in the teacher's work and how they can include information and collaboration between students.

Future work includes the design, development and deployment of a real use case where all the features of CAFCLA are implemented. This work will be 
developed by different teachers and developers in order to compare the results reached by all of them and evaluate the framework in a real scenario.

Acknowledgments. This project has been supported by the Spanish Ministry of Science and Innovation (Subprograma Torres Quevedo).

\section{References}

1. García, Ó., Tapia, D.I., Alonso, R.S., Rodríguez, S., Corchado, J.M.: Ambient intelligence and collaborative e-learning: a new definition model. Journal of Ambient Intelligence and Humanized Computing, 1-9 (2011)

2. Jorrín-Abellán, I.M., Stake, R.E.: Does Ubiquitous Learning Call for Ubiquitous Forms of Formal Evaluation?: An Evaluand oriented Responsive Evaluation Model. Ubiquitous Learning: An International Journal (2009)

3. Dey, A.K.: Understanding and Using Context. Personal and Ubiquitous Computing 5(1), 4-7 (2001)

4. Scardamalia, M., Bereiter, C., McLean, R.S., Swallow, J., Woodruff, E.: ComputerSupported Intentional Learning Environments. Journal of Educational Computing Research 5(1), 51-68 (1989)

5. Gómez-Sánchez, E., Bote-Lorenzo, M.L., Jorrín-Abellán, I.M., Vega-Gorgojo, G., Asensio-Pérez, J.I., Dimitriadis, Y.A.: Conceptual framework for design, technological support and evaluation of collaborative learning. International Journal of Engineering Education 25(3), 557-568 (2009)

6. Laine, T.H., Joy, M.S.: Survey on Context-Aware Pervasive Learning Environments. International Journal of Interactive Mobile Technologies 3(1), 70-76 (2009)

7. Bruce, B.C.: Ubiquitous learning, ubiquitous computing, and lived experience. In: Cope, W., Kalantzis, M. (eds.) Ubiquitous Learning, pp. 21-30. University of Illinois Press, Champaign (2008)

8. Chen, T.-S., Yu, G.-J., Chen, H.-J.: A framework of mobile context management for supporting context-aware environments in mobile ad hoc networks. In: Proceedings of the 2007 International Conference on Wireless Communications and Mobile Computing, pp. 647-652 (2007)

9. García, Ó., Alonso, R.S., Tapia, D.I., García, E., De la Prieta, F., de Luis, A.: CAFCLA: A conceptual framework to develop collaborative context-aware learning activities. In: Uden, L., Corchado, E.S., De Paz, J.F., De la Prieta, F. (eds.) Workshop on LTEC 2012. AISC, vol. 173, pp. 11-21. Springer, Heidelberg (2012)

10. Sharples, M., Taylor, J., Vavoula, G.: A Theory of Learning for the Mobile Age. In: Bachmair, B. (ed.) Medienbildung in neuen Kulturräumen, pp. 87-99. VS Verlag für Sozialwissenschaften, Wiesbaden (2010)

11. Glahn, C., Börner, D., Specht, M.: Mobile informal learning. In: Brown, E. (ed.) Education in the Wild: Contextual and Location-based Mobile Learning in Action, Nottingham. A report from the STELLAR Alpine Rendez-Vous Workshop series, pp. 28-31 (2010)

12. Neyem, A., Ochoa, S.F., Pino, J.A., Guerrero, L.A.: Sharing information resources in mobile ad-hoc networks. In: Fukś, H., Lukosch, S., Salgado, A.C. (eds.) CRIWG 2005. LNCS, vol. 3706, pp. 351-358. Springer, Heidelberg (2005) 
13. Hwang, G.-J., Yang, T.-C., Tsai, C.-C., Yang, S.J.H.: A context-aware ubiquitous learning environment for conducting complex science experiments. Computers \& Education 53(2), 402-413 (2009)

14. Blöckner, M., Danti, S., Forrai, J., Broll, G., De Luca, A.: Please touch the exhibits!: using NFC-based interaction for exploring a museum. In: Proceedings of the 11th International Conference on Human-Computer Interaction with Mobile Devices and Services, MobileHCI 2009, vol. 71, pp. 1-2 (2009)

15. Driver, C., Clarke, S.: An application framework for mobile, context-aware trails. Pervasive and Mobile Computing 4(5), 719-736 (2008)

16. Martín, S., Peire, J., Castro, M.: M2Learn: Towards a homogeneous vision of advanced mobile learning development. In: 2010 IEEE Education Engineering (EDUCON), pp. 569-574 (2010) 